\section{A Multistaged Fuzzy Logic Scheme in a Biobotanic Growth Regulation System}

\author{
Chung-Liang Chang ${ }^{1}$ and Ming-Fong Sie \\ Department of Biomechatronics Engineering, National Pingtung University \\ of Science and Technology, 91201, No. 1, Shuefu Road, Neipu, Pingtung \\ County, Taiwan, R.O.C.
}

Additional index words. fuzzy logic controller, expert system, crop growth control

\begin{abstract}
A multistaged fuzzy logic control method was used in the development of a bionic botanical growth control system. The growth mode combined fuzzy logic inference with expert knowledge to regulate the growth rate of plants. First, environment factors such as the light, temperature, and water required for plants in different stages of growth were analyzed. Fuzzy logic was then used to establish membership functions, an inference engine, and rule table. An expert database related to plant growth was combined with the fuzzy logic controller to formulate a plant growth control system. Sunflowers were used as a simulated model and the results correspond to the information provided by experts. The proposed model was used to control the growth rate of plants based on data provided in the expert database. The proposed method and results of this study are applicable in the management and control of environments for the growth of crops.
\end{abstract}

The process of plant growth is an extremely complex heterogeneous system. A growth model is used primarily to describe growth, differentiation, and development processes as well as to simulate the response of plants to the environment, thereby allowing quantitative analysis and dynamic control of the growth process of plants. Previous researchers divided plant growth models into empirical models and mechanism models (Thornley and France, 2007; Whisler et al., 1986). Empirical models, also called statistical models or correlation models, require clear descriptions of the causal relationships among variables. Mechanism models necessitate quantization before use based on physical or physiological behavior. In truth, the vast majority of crop growth models integrate both mechanisms with empirical models (Thornley and France, 2007) to coordinate theory and practice (Monteith, 1996).

Simple crop growth models require fewer variables, making them easier to use and understand and as a result, they are far more common. With ongoing development in computer technology, crop models are gradually becoming more complex. The Decision

Received for publication 22 Feb. 2012. Accepted for publication 15 Mar. 2012.

This work has been supported by the National Science Council, Taiwan, Republic of China, under grant NSC 101-2622-E-020-002-CC2.

Many thanks to the editor and reviewers for your precious comments to refine this article. We thank the National Science Council of Taiwan for their support of this work under grant 1012622-E-020-002-CC2.

${ }^{1}$ To whom reprint requests should be addressed; e-mail chungliang@mail.npust.edu.tw.
Support System for Agro-technology Transfer developed by the International Benchmark Sites Network for Agro-technology Transfer in the United States has effectively simulated the growth conditions of a number of crops (Acock and Acock, 1991; Engel et al., 1997; Jame and Cutforth, 1996). The concept of object orientation within this system was applied by van Evert and Campbell (1994) in the construction and management of an overall agriculture system, CropSys (Crop Systems). The concept has been extended to current expert systems, decision support systems, and artificial intelligence systems (van Evert et al., 2007). In recent years, many scholars have even integrated crop growth models with environment control models. In the future, crop growth models will eventually incorporate expert knowledge such as crop physiology, sensing technology, numerical analysis, and evolution analysis. However, to integrate and design a control system with a crop growth model can be difficult as a result of diversity of plant growth factors, comprising internal, external (environmental factors), and biological factors. Internal factors include the genetic and chromosomal characteristics of the plant as well as differences in plant growth regulators (PGRs). External factors include sunlight, temperature, humidity, water, nutrients, and gases. Biological factors include pathogenic microorganisms, pests, symbiotic microorganisms, and beneficial insects. All of these factors link to and restrict one another, the combined impact of which influences plant growth and development. of fuzzy set theory by Lotfi Zadeh (Zadeh, $1965,1972)$. This approach addresses the imprecise nature of control issues within all
Fuzzy logic began with the 1965 proposal physical systems. Nonetheless, to actually resolve problems requires that the target domain first be narrowed down. Experienced experts first share their knowledge of resolving these issues with a knowledge engineer, who then converts the professional knowledge into rules and writes them into a program. The system creates inferences according to these rules and provides less experienced users with a basis for their decisions. This computerized system, founded on a knowledge database, an inference engine, and an interface, is called an expert system. In recent years, fuzzy logic and expert decision programs have been widely adopted in smart control systems, covering home care, crop management, energy management, finances, and security monitoring (Fasanghari and Montazer, 2010; Kolhe et al., 2011; Waterman, 1986). However, conventional fuzzy expert systems must still deal with the problem of converting more complex problems into more complex expressions of expert knowledge. The number of fuzzy logic rules increases exponentially with the number of input variables and membership functions. As a result, typical single-layer fuzzy inference systems are insufficient for complicated systems with a large number of input variables. Plant growth models are particularly complex because the process of plant growth involves several stages, each with its own growth conditions and patterns. Appropriate growth rates in each stage have considerable influence on the quality of the plant after harvest. To address the issue of multiple variables and decisionmaking in botanical growth systems, plant growth must be dealt with in stages. Research has indicated that multilayer fuzzy inference can reduce the number of fuzzy rules $(\mathrm{Tu}$ et al., 2000). Kuo et al. (1998) developed a three-layer parallel fuzzy inference model to shorten the time of inference calculation in the fuzzy controller. Their enhanced fuzzy neural network is capable of distributed processing.

This study developed a multilayer parallel fuzzy inference system with the objective of establishing an expert system to monitor plant growth. The system features a reduced number of fuzzy rules, lower system complexity, and decreased calculation time. Sunflowers were used as the subject of simulation. An expert database regarding sunflower growth was constructed, the information of which could be used by the fuzzy inference engine to simulate the control of growth rate. Various environmental conditions are input into the initial parameter settings, and based on the varying environmental factors in each stage, the parameters can be set to approach the optimal growth curve of the particular plant. The contribution of this study lies in its capacity to clarify the proportions of environmental factors in each phase of growth through the inference of the proposed system. At the same time, each environmental factor is applied to complete the required growth in each stage within the allotted time, thereby enhancing planting quality.

The organization of this study is as follows: Chapter 2 describes the growth mechanisms 
of sunflowers and the structure of the proposed system. Chapter 3 presents the approach used to design the multilayer parallel fuzzy inference system. Chapter 4 exhibits the simulation and verification of the system, in which three scenarios were used to analyze and verify the system, and Chapter 4 presents our conclusions.

\section{Materials and Methods}

This study adopted sunflowers as the subject for growth simulation resulting from its wide applicability as a source of food, oil, animal feed, and biofuel (Putnam et al., 1990). In recent years, many experts have conducted research on methods to enhance production efficiency of sunflowers and to stabilize sunflower production. This study used a multilayer fuzzy inference engine in conjunction with expert knowledge to establish a model and control strategies for the growth of crops. To reduce the complexity

Table 1. Environmental factors.

\begin{tabular}{lcc}
\hline Sunlight & Temperature & Water (\%) \\
\hline $0 \mathrm{~h}$ & 0 to $-5^{\circ} \mathrm{C}$ & $0 \%$ \\
$1-4 \mathrm{~h}$ & $1-6^{\circ} \mathrm{C}$ & $10-20 \%$ \\
$4-7 \mathrm{~h}$ & $7-12{ }^{\circ} \mathrm{C}$ & $30-40 \%$ \\
$7-10 \mathrm{~h}$ & $13-24^{\circ} \mathrm{C}$ & $50-60 \%$ \\
$10-13 \mathrm{~h}$ & $25-28^{\circ} \mathrm{C}$ & $70-80 \%$ \\
$13-16 \mathrm{~h}$ & $29-33^{\circ} \mathrm{C}$ & $90-100 \%$ \\
Over $16 \mathrm{~h}$ & Over $34{ }^{\circ} \mathrm{C}$ & Over $100 \%$ \\
\hline
\end{tabular}

Table 2. Environmental factors required in each growth stage and corresponding growth rate.

\begin{tabular}{|c|c|c|c|c|c|c|c|c|c|}
\hline \multirow{3}{*}{$\frac{\text { Stage }}{\text { Stage }}$} & \multicolumn{2}{|c|}{ Sunlight } & \multirow{3}{*}{$\frac{\text { Temperature }}{\text { Degree }}$} & \multicolumn{2}{|r|}{ Water } & \multicolumn{2}{|c|}{ Growth ht } & \multicolumn{2}{|c|}{ Growth time } \\
\hline & & Cumulative & & & Cumulative & & Cumulative & & Cumulated \\
\hline & Hour & value & & $\mathrm{Kg}$ & value & $\mathrm{cm}$ & ht & Day & number of days \\
\hline$\overline{0}$ & 0 & 0 & 0 & 0 & 0 & 0 & 0 & 0 & 0 \\
\hline 1 & 0 & 0 & 4 & 2 & 2 & $1-4$ & $1-4$ & $1-2$ & $1-2$ \\
\hline 2 & $4-7$ & $4-7$ & 4.5 & 8 & 10 & $4-10$ & $5-14$ & $2-3$ & $3-5$ \\
\hline 3 & $10-13$ & $14-20$ & 8.5 & 25 & 35 & $10-18$ & $15-32$ & $4-6$ & $7-11$ \\
\hline 4 & $13-16$ & $27-36$ & 18 & 45 & 80 & $45-55$ & $60-87$ & $25-30$ & $32-41$ \\
\hline 5 & $10-13$ & $37-49$ & 26 & 200 & 280 & $30-38$ & $90-125$ & $10-20$ & $42-61$ \\
\hline 6 & $10-13$ & $47-62$ & 30 & 120 & 400 & $26-35$ & $126-160$ & $35-55$ & $77-111$ \\
\hline 7 & $7-10$ & $54-72$ & 2 & 0 & 400 & $0-10$ & $126-170$ & $3-5$ & $80-116$ \\
\hline
\end{tabular}

of analysis using the model, this study precluded the influence of internal and biological factors, varying only the sunlight, temperature, and water to control the growth of the plants. Even the impact of parent plants on seed quality was disregarded.

Sunflower growth description. In accordance with the literature, the growth process of sunflowers was divided into eight stages and marked the approximate range of time required for each stage (Cook, 2009b; Marks, 1994; Meyer et al., 1999; Schneiter and Miller, 1981). The 0th stage is the seed stage (point of origin); the first stage is infancy (1 to $2 \mathrm{~d}$ ) followed by germination to cotyledons ( 1 to $3 \mathrm{~d}$ ), cotyledons to leaf ( 4 to $6 \mathrm{~d}$ ), leaf to appearance of buds ( 25 to $30 \mathrm{~d}$ ), appearance of buds to flowering (10 to $20 \mathrm{~d}$ ), flowering to maturity ( 25 to $55 \mathrm{~d}$ ), and finally, wither/ harvest ( 3 to $5 \mathrm{~d}$ ). The optimal environmental factors of each stage vary; therefore, this study selected three environmental factors: sunlight, temperature, and water, and divided them into several levels, as shown in Table 1. Among the factors, sunflowers require $\approx 350$ to $500 \mathrm{~kg}$ of water in total during the growth process. In terms of the quantities required in each stage, previous studies stated that the maximum amount of water in each stage may not exceed $50 \%$ of the total. Therefore, the total amount of required amount of water was set as $400 \mathrm{~kg}$ and $50 \%(200 \mathrm{~kg})$ as the maximum amount per stage. In Table 1, 50\% indicates $100 \mathrm{~kg}$ of water is required.
Table 2 presents the environmental factors required by sunflowers in each stage of growth as well as the corresponding growth height and growth time. As seen in the table, environmental factors indeed influence plant growth height and growth time, even without considering the generic characteristics of the plant itself. To simplify analysis and facilitate the design of the growth regulation system, this study divided the plant growth process into stages according to the number of environmental factors influencing growth height and growth time. Excluding the stage of wither, seven stages were established, from 0 to 6 .

Proposed system description. This study combined multilayered fuzzy controllers to form a botanical growth regulation system for the complete growth cycles of plants (Chang and Sie, 2011). This design was adopted because the environmental conditions that plants require in each stage of growth vary greatly and setting all the required environmental conditions within one fuzzy controller would generate a high degree of complexity when defining the membership functions, fuzzy sets, and fuzzy rules. Designing a fuzzy controller for each stage significantly decreases the overall design complexity of the system. The multilayers and fuzzy control system proposed in this study consist of two parts: a fuzzy main controller and several fuzzy subcontrollers. The main controller is a single fuzzy controller, whereas the subcontrollers are divided into two groups according to growth phase, each containing eight fuzzy controllers. During each stage of growth, only one controller in either group is used. The overall framework of the multilayered fuzzy control system is shown in Figure 1. The procedure of this system is as follows:

Step 1: The initial values of sunlight, temperature, and water for each growth stage are entered into the input port. Because plant growth regulation system is very complex, the most basic environment

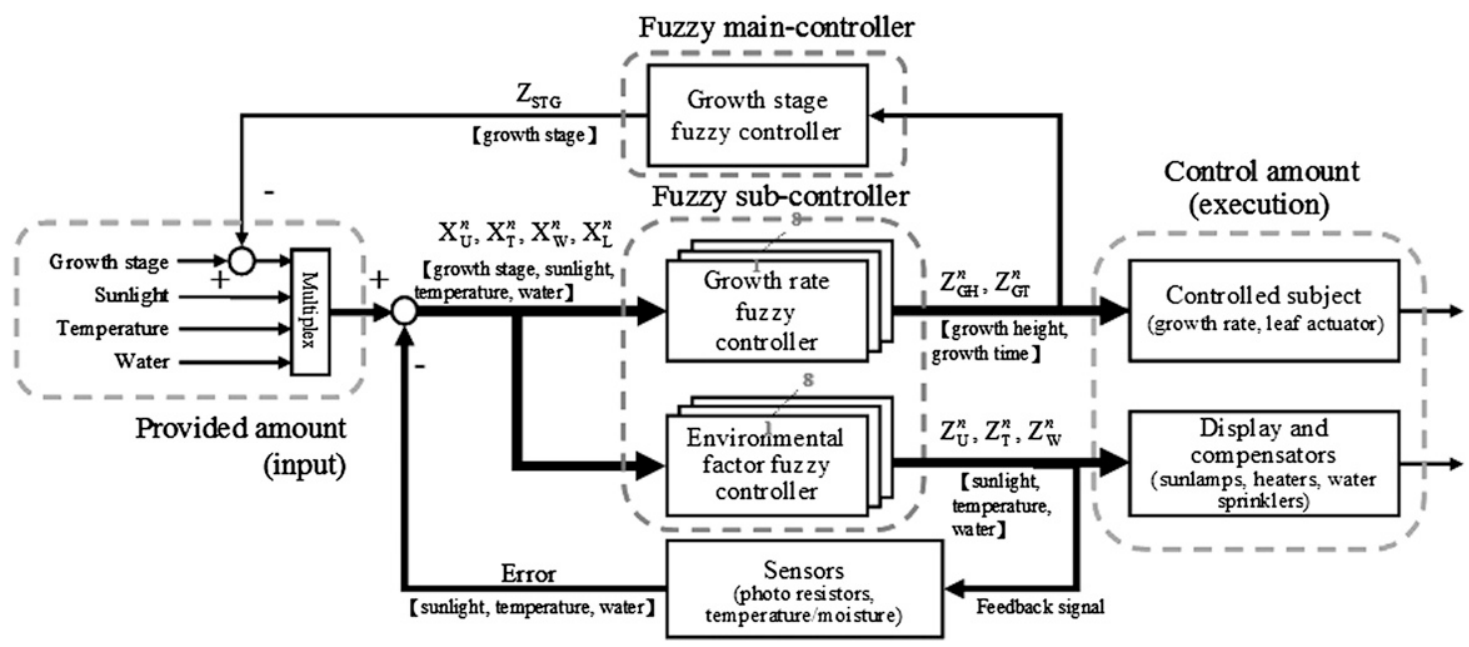

Fig. 1. Framework of the multilayered and fuzzy system. 
Table 3. Input values and linguistic labels.

\begin{tabular}{|c|c|c|c|c|c|c|c|c|}
\hline \multicolumn{3}{|c|}{$\mathrm{X}_{\mathrm{L}}^{n}$} & \multicolumn{2}{|c|}{$\mathrm{X}_{\mathrm{U}}^{n}$} & \multicolumn{2}{|c|}{$\mathrm{X}_{\mathrm{T}}^{n}$} & \multicolumn{2}{|c|}{$\mathrm{X}_{\mathrm{W}}^{n}$} \\
\hline Stage $n$ & {$\left[a_{\mathrm{L}}^{n}, b_{\mathrm{L}}^{n}, c_{\mathrm{L}}^{n}\right]$} & Linguistic labels & {$\left[a_{\mathrm{U}}^{n}, b_{\mathrm{U}}^{n}, c_{\mathrm{U}}^{n}\right]$} & Linguistic labels & {$\left[a_{\mathrm{T}}^{n}, b_{\mathrm{T}}^{n}, c_{\mathrm{T}}^{n}\right]$} & Linguistic labels & {$\left[a_{\mathrm{W}}^{n}, b_{\mathrm{W},}^{n}, c_{\mathrm{W}}^{n}\right]$} & Linguistic labels \\
\hline 1 & {$[1,2,3]$} & LL, LM, LH & {$[0,0.5,1]$} & SM, SH, SVH & {$[-5,4,13]$} & TL, TM, TH & {$[0,2,4]$} & WL, WM, WH \\
\hline 4 & {$[4,5,6]$} & LL, LM, LH & {$[13,14.5,16]$} & SL, SM, SH & {$[-5,18,41]$} & TL, TM, TH & {$[0,45,90]$} & WL, WM, WH \\
\hline 5 & {$[5,6,7]$} & LL, LM, LH & {$[10,11.5,13]$} & SL, SM, SH & {$[-5,26,57]$} & TL, TM, TH & {$[0,200,400]$} & WL, WM, WH \\
\hline 6 & {$[6,7,8]$} & LL, LM, LH & {$[10,11.5,13]$} & SL, SM, SH & {$[-5,30,65]$} & TL, TM, TH & {$[0,120,240]$} & WL, WM, WH \\
\hline
\end{tabular}

factors such as sunlight, water, and temperature are used to regulate plant growth without considering such uncertainties as disease, insect pest, and such human factors as PGR agent, plant hormone, fertilizer, carbon dioxide, etc.

Step 2: The initial values are sent to the subcontrollers for growth rate and environmental factors selected by the main controller based on growth stage. Growth rate and growth time are regulated according to the output of the growth rate subcontrollers; the output signal of the environment factor subcontrollers is fed back into the input port to generate an error signal, which is resent into the subcontrollers to adjust regulation.

Step 3: The output signal of the growth rate fuzzy controller is fed back to the main controller to determine the current growth stage and generate another output signal, which is subtracted from the signal of the output port to generate an error signal. This error signal is then sent into the subcontrollers to adjust regulation.

The system described is a self-adjusting system. Once the initial values are input into the system, the system regulates plant growth. The following section presents the design approach of the fuzzy controllers in Figure 1.

The steps involved in designing the proposed system are as follows:

Step 1: Based on the eight growth stages identified in this study, the proportions of individual environmental factors were obtained from literature and the experience of experts to establish environmental parameters suitable for each stage of growth (Allinne et al., 2009; Unger, 1986).

Step 2: A bionic botanical growth fuzzy controller was produced, comprising a fuzzy main controller and subcontrollers.

Step 3: The results obtained by the fuzzy controller were compared with data from the literature.

In general, fuzzy controllers are designed in the following order: 1) definition of input variables, output variables, and linguistic variable database; 2 ) determination of fuzzification strategies; 3) design of controlling rule database; 4) develop methods of fuzzy inference; and 5) defuzzification.

In the following, our design approach is described and introduced.
Table 4. Output values and linguistic labels.

\begin{tabular}{lccccccc}
\hline & \multicolumn{4}{c}{$\mathrm{Z}_{\mathrm{GH}}^{n}$} & & \multicolumn{3}{c}{$\mathrm{Z}_{\mathrm{GT}}^{n}$} \\
\cline { 2 - 4 } Stage $n$ & Truth value & {$\left[a_{\mathrm{GH}}^{n}, b_{\mathrm{GH}}^{n}, c_{\mathrm{GH}}^{n}\right]$} & Linguistic labels & & Truth value & {$\left[a_{\mathrm{GT}}^{n}, b_{\mathrm{GT}}^{n}, c_{\mathrm{GT}}^{n}\right]$} & Linguistic labels \\
\hline 0 & 0 & {$[0,0.5,1]$} & HM, HH, HVH & & 0 & {$[0,2.5,5]$} & GTM, GTH, GTVH \\
1 & $1-4$ & {$[0,0.5,1]$} & HVL, HL, HM & & $1-2$ & {$[0,1,2]$} & GTL, GTM, GTH \\
2 & $5-14$ & {$[0,1.5,3]$} & HVL, HL, HM & & $2-3$ & {$[0,3,6]$} & GTL, GTM, GTH \\
3 & $15-32$ & {$[0,3,6]$} & HVL, HL, HM & & $4-6$ & {$[0,6,18]$} & GTL, GTM, GSH \\
4 & $60-87$ & {$[0,5,10]$} & HVL, HL, HM & & $25-30$ & {$[0,30,60]$} & GTL, GTM, GTH \\
5 & $90-125$ & {$[0,12.5,25]$} & HVL, HL, HM & & $10-20$ & {$[0,20,40]$} & GTL, GTM, GTH \\
6 & $126-160$ & {$[0,15,30]$} & HVL, HL, HM & & $35-55$ & {$[0,55,110]$} & GTL, GTM, GTH \\
7 & $126-170$ & {$[0,15,30]$} & HVL, HL, HM & $3-5$ & {$[0,5,10]$} & GTL, GTM, GTH \\
\hline
\end{tabular}

Definition of input variables, output variables, and linguistic variable database. The multilayered and fuzzy control system comprises eight subcontrollers dealing with environmental factors, eight subcontrollers for growth rate, and one main controller for the stages of growth. Input variables and output variables are defined subsequently.

A. Input variables: 1. Fuzzy subcontroller for environmental factors and growth rate:

The number of input variables for environmental factors and growth rate are the same. In the $n$th stage, the input variables are SUNLIGHT $\left(\mathrm{X}_{\mathrm{U}}^{n}\right)$, TEMPERATURE $\left(\mathrm{X}_{\mathrm{T}}^{n}\right)$, and WATER $\left(X_{\mathrm{W}}^{n}\right)$. Fuzzy domains were established based on proportions described in the literature as well as three fuzzy sets (Marks, 1994). The linguistic statements were: VERY LOW (VL), LOW (S), MEDIUM (M), HIGH $(\mathrm{H})$, and VERY HIGH (VH), among which three were selected to establish the fuzzy sets. To determine the current stage of growth, another input variable was added: LEVEL $\left(\mathrm{X}_{\mathrm{L}}^{n}\right)$. Subsequently, the input variables and fuzzy set statements for each stage are introduced.

(a) $\mathrm{X}_{\mathrm{U}}^{n}$ : At $n=2,3, \ldots, 7$, the linguistic statements for each stage are SUNLIGHT LOW (SL), SUNLIGHT MEDIUM (SM), and SUNLIGHT HIGH (SH). At $n=0,1$, the three fuzzy sets are SUNLIGHT MEDIUM (SM), SUNLIGHT HIGH (SH), and SUNLIGHT VERY HIGH (SVH). In the eight stages, there are a total of 24 fuzzy sets for sunlight.

(b) $\mathrm{X}_{\mathrm{T}}^{n}$ : At $n=0,1,2, \ldots, 7$, the fuzzy sets are TEMPERATURE LOW (TL), TEMPERATURE MEDIUM (TM), and TEMPERATURE HIGH (TH). In the eight stages, there are a total of 24 fuzzy sets for temperature.

(c) $\mathrm{X}_{\mathrm{W}}^{n}$ : At $n=2, \ldots, 6$, the three fuzzy sets are WATER LOW (WL), WATER

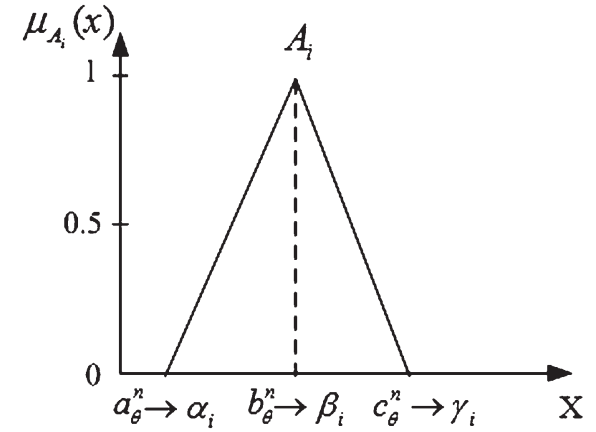

Fig. 2. Membership function of fuzzification.

MEDIUM (WM), and WATER HIGH (WH). At $n=1,7$, the three fuzzy sets are WATER MEDIUM (WM), WATER HIGH (WH), and WATER VERY HIGH (WVH). In the eight stages, there are a total of 24 fuzzy sets for water.

(d) $\mathrm{X}_{\mathrm{L}}^{n}$ : At $n=0,1,2, \ldots 6$, the three fuzzy sets are LEVEL LOW (LL), LEVEL MEDIUM (LM), and LEVEL HIGH (LH). fuzzy sets for level, among which the level setting for the eighth stage enables a return to the first stage (the 0th stage) at the end.

2. Growth stage main controller:

The input port of the growth stage main controller can be regarded as the output ports of the growth rate subcontrollers. That is to say, the output variables of the growth rate subcontrollers become the input variables of the main controller. In growth height $\left(\mathrm{X}_{\mathrm{GH}}^{n}\right)$, the input variables include eight fuzzy sets, each of which include: GH0, GH1, GH2, GH3, GH4, GH5, GH6, and GH7; in growth time $\left(\mathrm{X}_{\mathrm{GT}}^{n}\right)$, there are also eight fuzzy sets, each of which include: GT0, GT1, GT2, GT3, GT4, GT5, GT6, and GT7. In the eight stages, there are a total of 24 
A

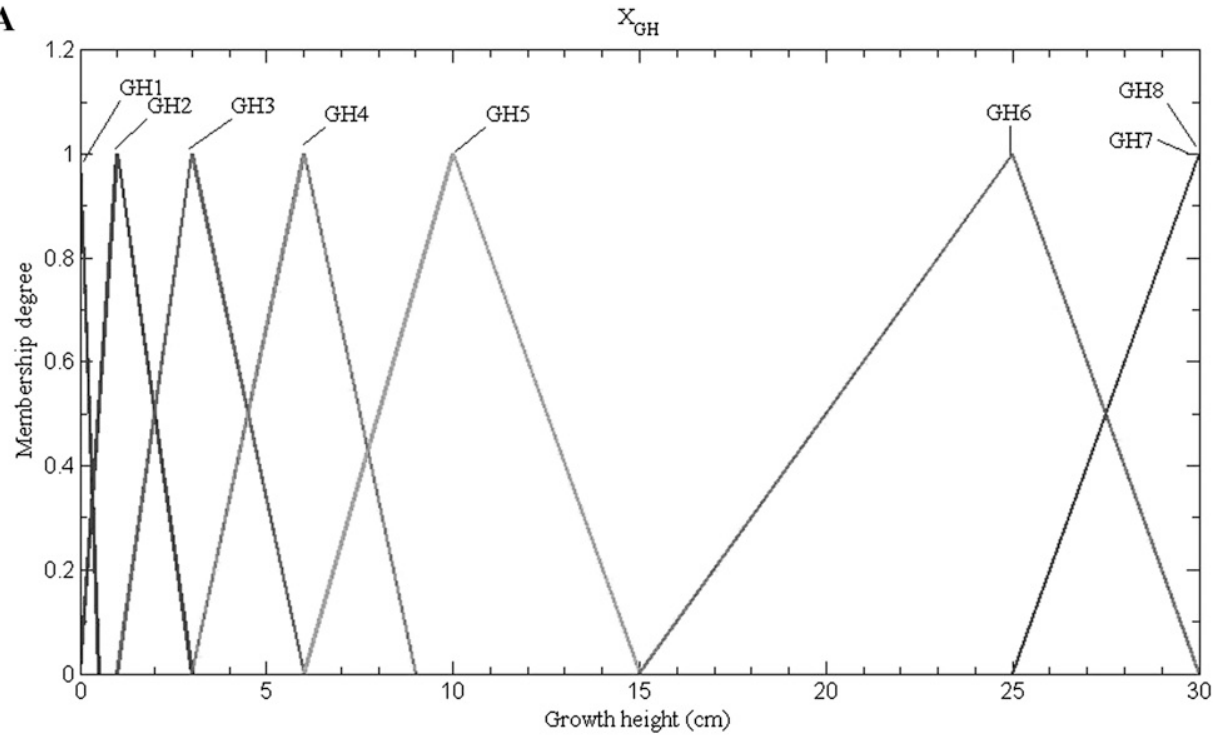

B

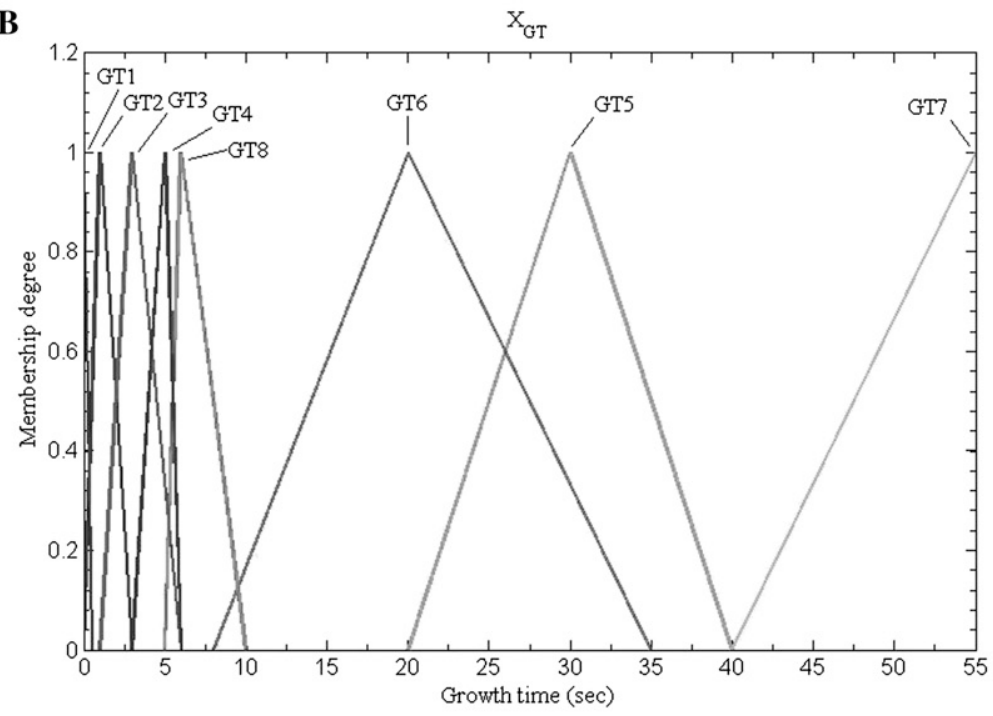

C

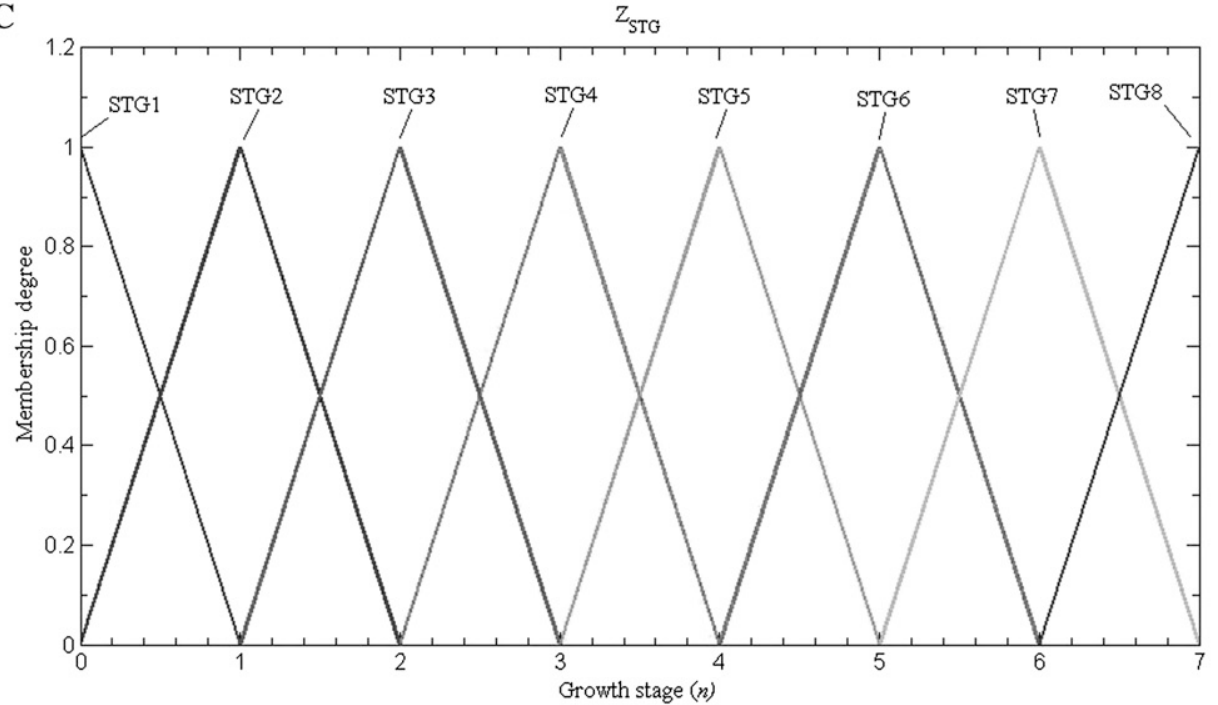

Fig. 3. Input and output membership functions of growth stage controller; (A) membership function of GH fuzzification; (B) membership function of GT fuzzification; (C) membership function of growth stage controller output.

The primary purpose of the main controller is to determine whether the output values of the growth rate fuzzy subcontrollers meet the conditions for the next stage.
B. Output variables: 1 . Environmental factor fuzzy subcontrollers:

The output variables of the environmental fuzzy subcontrollers are sunlight $\left(Z_{\mathrm{U}}^{n}\right)$, temperature $\left(\mathrm{Z}_{\mathrm{T}}^{n}\right)$, and water $\left(\mathrm{Z}_{\mathrm{W}}^{n}\right)$. Their main purpose is to provide compensation for environmental factors. When too much is provided, supply is reduced; when supply 


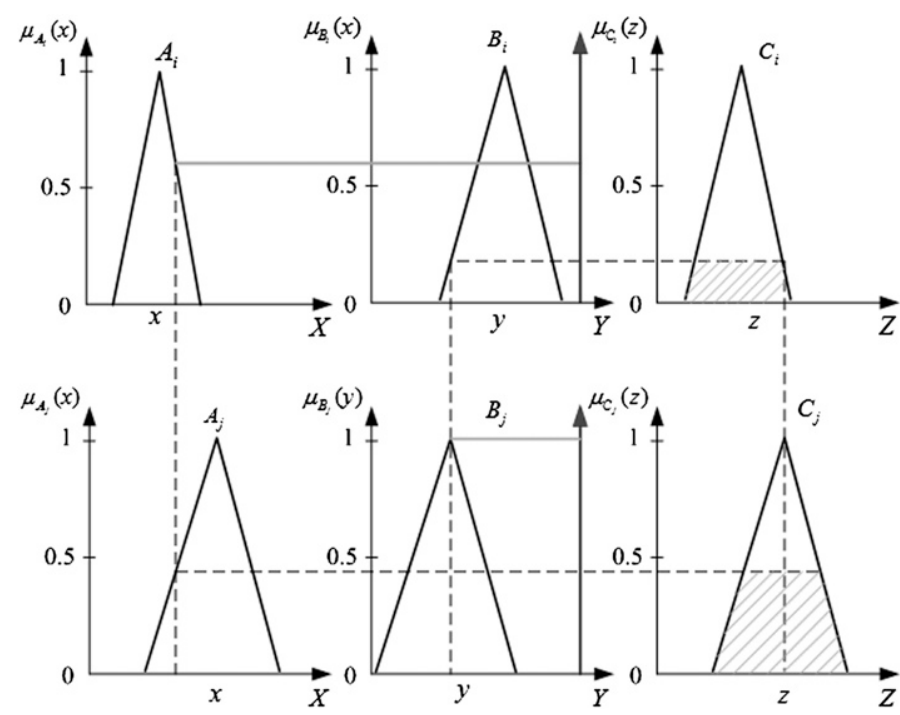

Fig. 4. Product method of fuzzy inference.

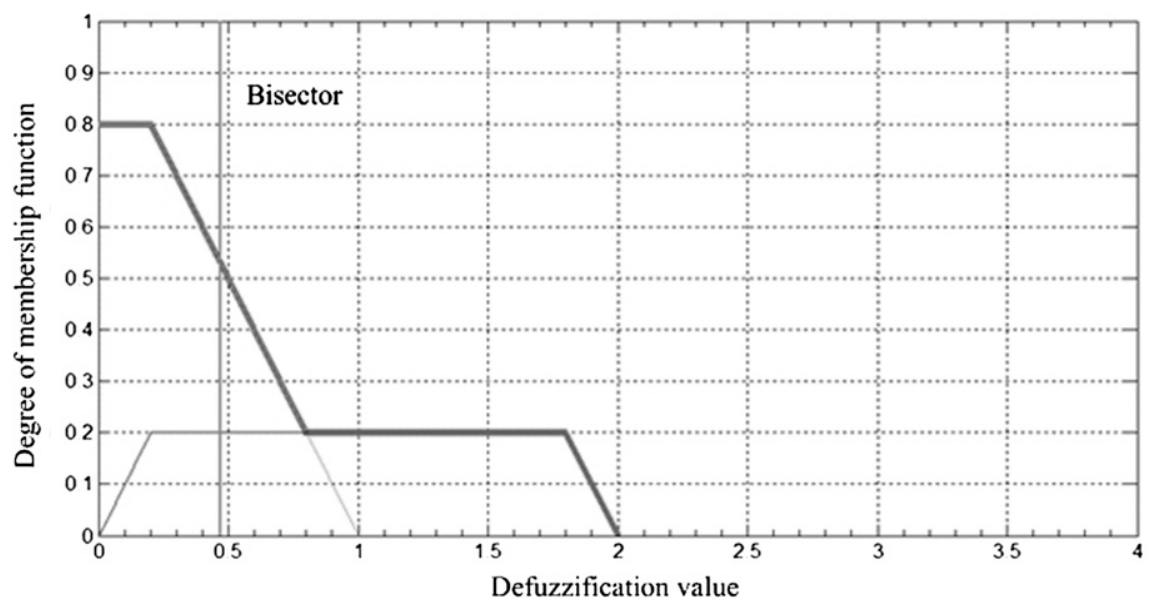

Fig. 5. Defuzzification value derived using the bisector method.

falls short, more is provided. These variables were also divided into eight stages, each with three fuzzy sets. The design is the same as that of the input variables.

2. Growth rate fuzzy subcontrollers:

The output variables for these subcontrollers include growth height $\left(\mathrm{Z}_{\mathrm{GH}}^{n}\right)$ and growth time $\left(Z_{\mathrm{GT}}^{n}\right)$.

(a) $\mathrm{Z}_{\mathrm{GH}}^{n}$ :

According to past studies (Cook, 2009a. 2009b; Meyer et al., 1999), the height of sunflowers averages between 100 and $200 \mathrm{~cm}$ with some plants surpassing $300 \mathrm{~cm}$. On the same principle, the proportion of height was shrunk to $30 \mathrm{~cm}$ and defined the appropriate growth height for each stage. At $n=1,2 \ldots, 7$, the three fuzzy sets are VERY LOW (VL), LOW (L), and MEDIUM (M). At $n=0$, the three fuzzy sets are MEDIUM (M), HIGH (H), and VERY HIGH (VH). The reason for this setting was to enable a return to $0 \mathrm{~cm}$ in the first stage. Therefore, in the eight stages, there are a total of 24 fuzzy sets. (b) $\mathrm{Z}_{\mathrm{GT}}^{n}$ :

According to the same sources stated previously, the time required for sunflowers to develop from seed to maturity is generally 90 to $120 \mathrm{~d}$. The length of that period depends on the varietal, sowing period, and cultivation environment. The complete development was reduced using a ratio from $120 \mathrm{~d}$ to $120 \mathrm{~s}$ to enable simulation of the entire development cycle within a short time. At $n=1,2 \ldots, 7$, the three fuzzy sets are LOW (L), MEDIUM $(\mathrm{M})$, and HIGH $(\mathrm{H})$. At $n=0$, the three fuzzy sets are MEDIUM (M), HIGH (H), and VERY $\mathrm{HIGH}(\mathrm{VH})$. The reason for this setting was to enable a return to $0 \mathrm{~cm}$ in the first stage. In the eight stages, there are a total of 24 fuzzy sets.

3. Growth stage main controller:

The primary purpose of the growth stage main controller is to act as a decision-making system determining whether the next stage can be reached from the output of the growth rate fuzzy subcontrollers. The main controller has only one growth stage output variable $\left(Z_{\mathrm{STG}}\right)$ with eight output fuzzy sets: STG0,

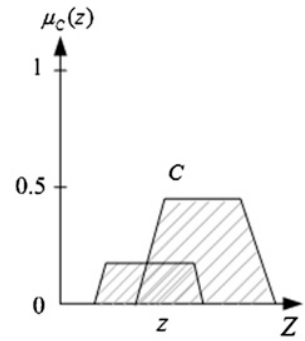

STG1, STG2, STG3, STG4, STG5, STG6, and STG7.

The input variables, output variables, and fuzzy linguistic labels are organized in Tables 3 and 4 .

Within the tables, $a_{\theta}^{n}, b_{\theta}^{n}$, and $c_{\theta}^{n}$ represent the physical values corresponding to the three vertexes of the triangular membership function; $\theta$ represents any of the various input variables; and $n$ is the current stage. The design of the fuzzy membership function is described in the next section.

As mentioned previously, the subcontrollers for each stage use four input variables and two output variables with three fuzzy sets to each variable. Thus, there are a total of $4 \times$ $3 \times 8=96$ input fuzzy sets and $2 \times 3 \times 8=48$ output fuzzy sets in the entire fuzzy subcontroller system. The main controller has a total of $2 \times 8=16$ input fuzzy sets and $1 \times 8=$ eight output fuzzy sets.

Determination of fuzzification strategies. Once the settings for the variables and fuzzy sets were complete, fuzzification strategies could be determined. The appropriately proportioned input and output variables were first converted into the fuzzy domain before the membership function is selected and fuzzificated followed by the corresponding relative membership degree. This study selected a triangle membership function (trimf). Regarding the selection type of membership function, bell-shaped, trapezoidal, single tone typed are adopted. However, the use of a triangular type presents better performance. Thus, the triangular type is used as the membership function for controller. The function $\mu_{A_{i}}(x)$ is

$\mu_{A_{i}}(x)=\left\{\begin{array}{cc}0, & x<\alpha_{i} \\ \frac{x-\alpha_{i}}{\beta_{i}-\alpha_{i}}, & \alpha_{i} \leq x \leq \beta_{i} \\ \frac{\gamma_{i}-x}{\gamma_{i}-\beta_{i}}, & \beta_{i} \leq x \leq \gamma_{i} \\ 0, & x>\gamma_{i}\end{array}\right.$.

where $A_{i}$ represents the $i$ th fuzzy set, and the values of $\alpha_{i}, \beta_{i}$, and $\gamma_{i}$ depend on the 


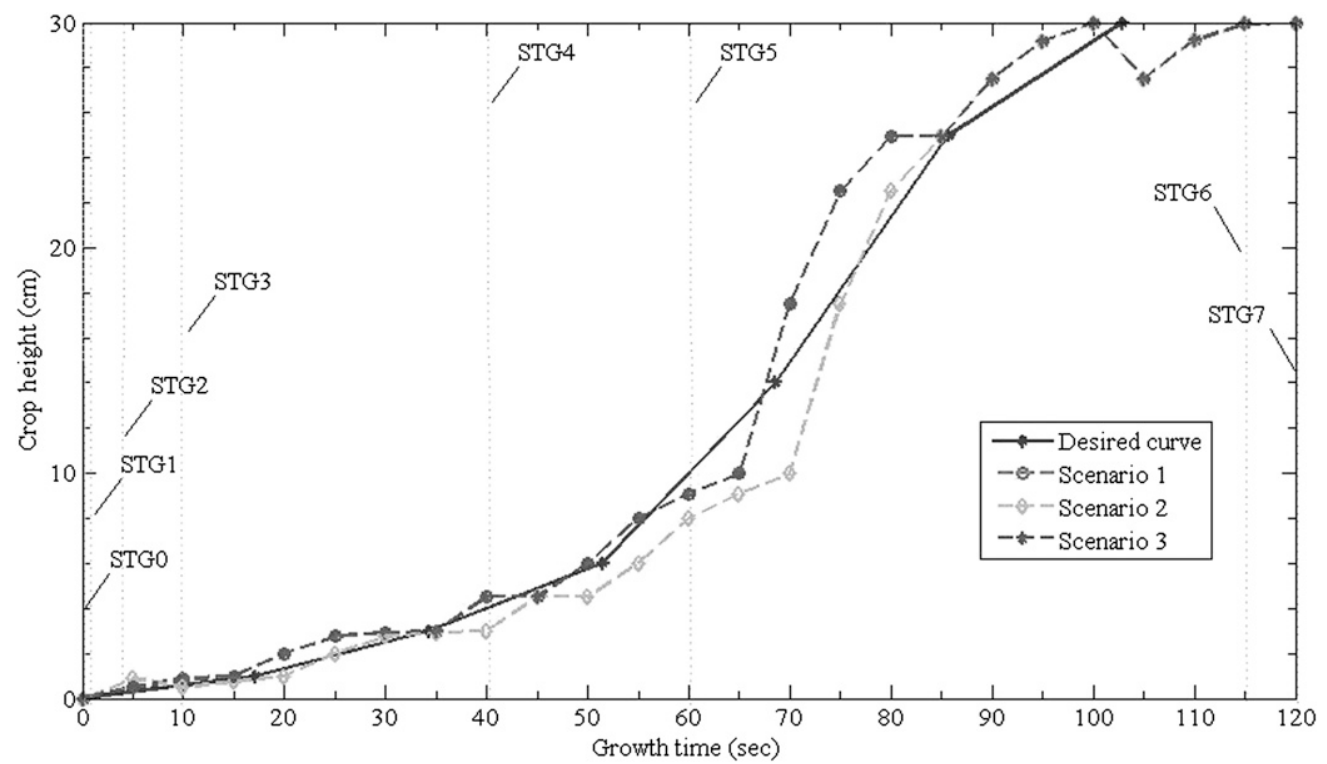

Fig. 6. Comparison of expected values and fuzzy control simulation results for growth height.

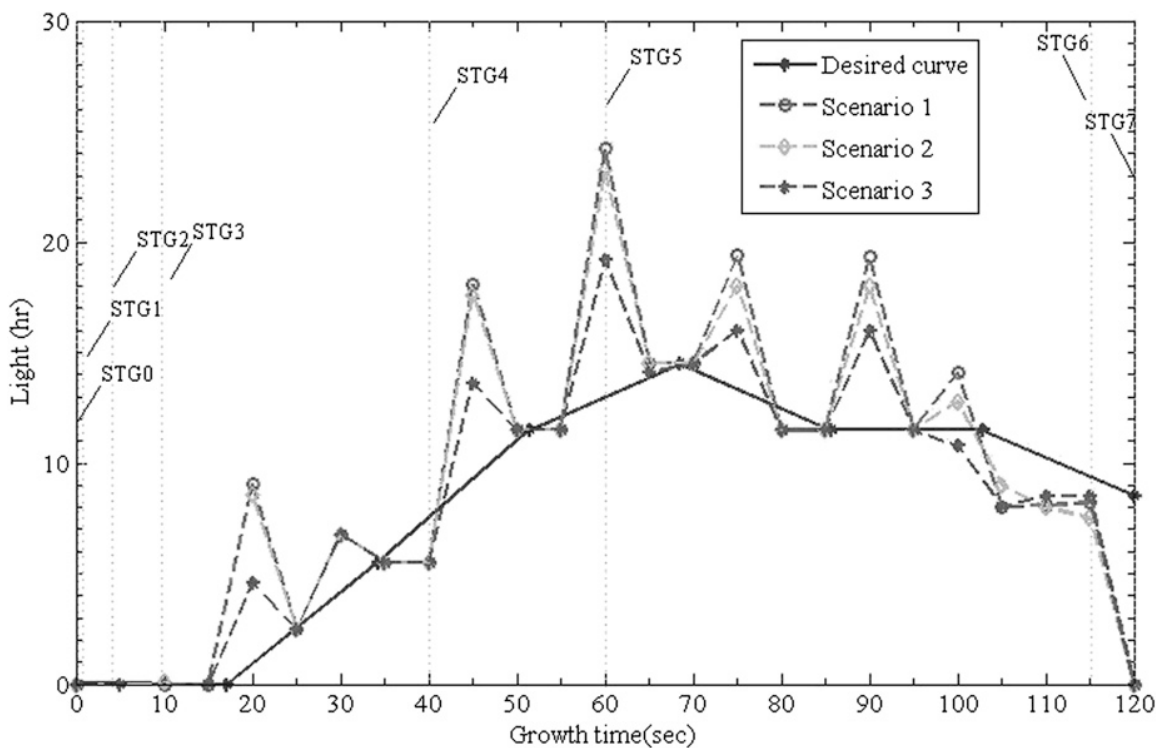

Fig. 7. Comparison of expected values and fuzzy control simulation results for sunlight.

linguistic labels. Following is the execution of fuzzification, a crucial procedure in which input data are converted into fuzzy numbers using the membership function. When a specific value is entered, it is calculated with the predesigned membership function using an intersection operation to derive the degree of membership, the result of fuzzification. Figure 2 depicts the defined items in the membership function. Figure 3 exhibits the distributions of the input and output membership functions in the main controller.

Design of fuzzy rules database. The inference rules in this study were established in accordance with the order of development of the input variables, output variables, and linguistic labels (Tables 3 and 4). The rules were formulated taking into full consideration the accumulated experience of experts and information from past studies. In addition, the proposed system contains multilayer fuzzy controllers divided into the growth stage main controller and the subcontrollers for growth rate and environmental factors. As a result of differences in objectives, the design of the controllers differed slightly. The definitions of the rules in the main controller database were simpler; operations only required the determination of growth stages according to growth time and growth height. The rule databases of the subcontrollers for growth time and environmental factors, one for each stage, were divided into three level distinctions: LOW, MEDIUM, and HIGH as the main judgment cores. The relevant designs of the rule databases are introduced in Appendix A.

Appendix A shows that when the current stage is STG0, the expected output value is
STG1. When the current level is at LOW, the output must be increased to achieve the expected output value. In contrast, when the level is at HIGH, the output value must be decreased to achieve the expected output. At MEDIUM, the output is the same. The fuzzy rules database for each stage contains 29 rules, which are the core rules of the control system. In addition to the eight stages in the main controller, a total of 232 rules constitutes the bionic botanical growth control system. The rules are expressed in the form of "If... and...then..." For example, in a circumstance of LEVEL LOW, the $k$ th rule may look like the following:

LS:

Rule $k$ : If $\mathrm{X}_{\mathrm{U}}$ is $\mathbf{U L} A N D \mathrm{X}_{\mathrm{T}}$ is TL $A N D$ $\mathrm{X}_{\mathrm{W}}$ is WL Then $\mathrm{Z}_{\mathrm{GH}}$ is $\mathbf{G H H}$ AND $\mathrm{Z}_{\mathrm{GT}}$ is GTH

When the current situation shows LEVEL LOW (LL) (for example, sunlight or water is insufficient; the temperature is too low), the settings for growth height and time are increased.

Method of fuzzy inference. Within any single inference rule, the intersection condition AND is calculated using the Max-Dot method, as shown in Figure 4, where $\mu_{A i}^{n}$ and $\mu_{B i}^{n}$ are the respective $\mu$ membership degrees of $A$ and $B$ in the $i$ th rule of the $n$th stage. Similarly, $\mu_{A j}^{n}$ and $\mu_{B j}^{n}$ are the respective $\mu$ membership degrees of $A$ and $B$ in the $j$ th rule of the $n$th stage. $\mu_{C}$ is the degree of membership of the output variables; $A$ and $B$ represent the input fuzzy sets, and $C$ is the output fuzzy set; $x_{0}$ and $y_{0}$ are the element values of the input fuzzy subsets, and the rules are linked by a union operation.

Defuzzification. Fuzzy inference results in the output of fuzzy sets. The identification of a specific value to represent from the fuzzy set domain is called defuzzification. A simpler approach is the bisector method, which first obtains the area of the region enclosed by the membership function curve of the fuzzy 
sets and vertical coordinates before identifying a vertical line that bisects the region into two portions of equal area (see Fig. 5). The coordinate of this line on the $\mathrm{X}$-axis is the resulting defuzzification value. Supposing the membership function of fuzzy set $A$ in domain $U$ is $A(u), u \in U$, and the horizontal coordinate of the horizontal line bisecting the region surrounded by the membership function curve and the horizontal coordinates is $u_{\text {bis }}$, let $u \in[a, b]$, where a,b are known constants. From the differentiation of Eq. (2), $u_{b i s}$ can be derived as:

$$
\begin{aligned}
\int_{\mathrm{a}}^{u_{b i s}} A(u) d u & =\int_{u_{b i s}}^{b} A(u) d u \\
& =\frac{1}{2} \int_{a}^{b} A(u) d u
\end{aligned}
$$

\section{Simulation Results}

This chapter demonstrates the use of the multilayer fuzzy controller designed in this study to control growth rates and conditions as anticipated by the expert knowledge database. Three simulation scenarios with different parameter settings at the seed stage (STG0) were implemented. The output results from the controllers were compared with the expected values in the expert knowledge database.

Parameter settings for growth model. The parameters for the growth mode are the initial settings provided before simulation. Different settings allowed observation of changes in growth rate and environmental factors regulated by the fuzzy controllers.

The settings for the three simulation scenarios are as follows.

(A) Scenario 1: The level setting for STG1 was stipulated as LL, and the initial settings for $\mathrm{X}_{\mathrm{U}}^{1}, \mathrm{X}_{\mathrm{T}}^{1}$, and $\mathrm{X}_{\mathrm{W}}^{1}$ were all 1 . The reason behind these settings was that the decisions made by the rules in the fuzzy rule database were designed to weigh the STG level. The STG1 in this simulation was expected to develop to STG2, hence the LL setting. This allows observation of whether this setting increases growth rate to approach that of the expected values in STG1.

(B) Scenario 2: The level setting for STG1 in this scenario was stipulated as LM, and the initial settings for $X_{U}^{1}, X_{T}^{1}$, and $X_{W}^{1}$ were all 1. The STG1 in this simulation was expected to develop to STG2; therefore, the setting of LM should be appropriate. This allows observation of whether the growth rate resulting from this setting conforms to expected values in STG2.

(C) Scenario 3: The level setting for STG1 in this scenario was stipulated as LH, and the initial settings for $\mathrm{X}_{\mathrm{U}}^{1}, \mathrm{X}_{\mathrm{T}}^{1}$, and $\mathrm{X}_{\mathrm{W}}^{1}$ were all 2. The STG1 in this simulation was expected to develop to STG2. This allows observation of whether this setting increases growth rate gradually to approach that of expected values.
Verification of software loop. This study constructed simulation using MATLAB/ Simulink. Using the proposed fuzzy control system, the capability of the bionic botanical growth system was investigated to conform to the values from the expert knowledge database and reach stability.

To determine whether the control system is able to regulate plant growth to approach expected values even with parameter settings different from those in the knowledge database, simulation scenarios with three different initial settings were conducted for analysis.

Figure 6 compares the expected values and the simulation results for growth height. Figures 7, 8, and 9 compare the results of the three simulations with the expected values for sunlight, temperature, and water. These figures illustrate that the amount of environment factor presents a sudden increase. The occurrence of such phenomenon is because during the plant growth process, the initial value of such factors as sunlight, water, and temperature in each stage is not the same. Thus, the sudden increase of these factors is a good opportunity to test system performance. The curve demonstrates that the value of each factor converges to desired curve.

Analysis of system complexity. In general, the greatest number of design rules equals the number of input variables and that of output variables, respectively, multiplied by the number of fuzzy sets:

$$
\Re=\Pi_{\mathrm{X}}^{p} \times \Pi_{\mathrm{Z}}^{q}
$$

where $\Re$ is the number of rules; $\Pi_{X}$ represents the number of input sets, and $\Pi_{Z}$ represents the number of output sets; $p$ and $q$ are the numbers of input and output variables. According to the system designed in

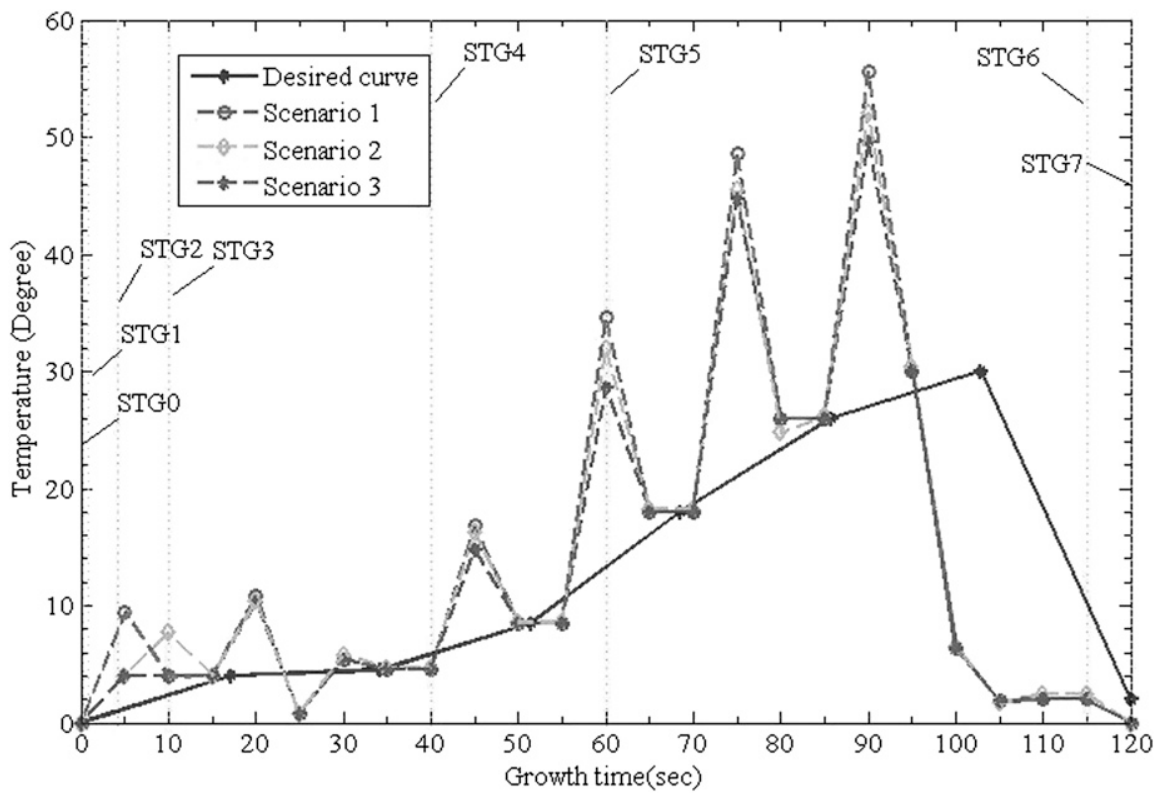

Fig. 8. Comparison of expected values and fuzzy control simulation results for temperature.

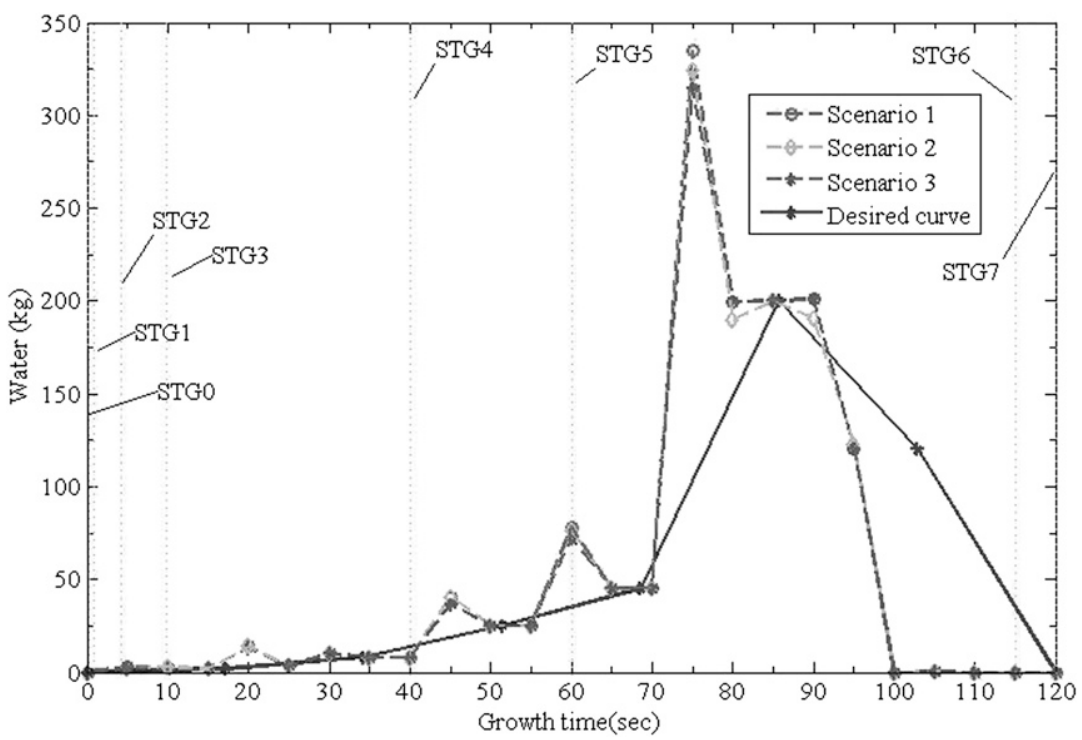

Fig. 9. Comparison of expected values and fuzzy control simulation results for water. 
this study, each growth rate controller has a total of four input variables and two output variables with three fuzzy sets each. Substituted into Eq. (3), a total of 729 rules were gained. However, this study achieved ideal initial control with only 29 rules. In terms of execution speed, this study divided the subsystem into two groups, each with eight fuzzy controllers. Two controllers, one in each group, are operated simultaneously; the parallel and scattered design therefore enables greater speed than that of a single large controller.

\section{Conclusion}

This study developed a multilayer parallel fuzzy inference device to construct a bionic botanical growth control system. Using fuzzy inference, this system accurately identified the required environmental parameters. In circumstances in which the values were sufficient, plants were able to achieve the designated growth height within the regulated time. To deal with system complexity, this study used staging and parallel designs to reduce difficulty in hardware implementation and increase execution speed. Only external factors (environmental factors) of plant growth were considered. It is suggested that future studies consider internal factors influencing botanical growth to provide more comprehensive control and management. The system developed in this study can be used in automated plant cultivation systems.

\section{Literature Cited}

Acock, B. and M.C. Acock. 1991. Potential for using long-term field research data to develop and validate crop simulators. Agron. J. 83:56-61.
Allinne, C., P. Maury, A. Sarrafi, and P. Grieu. 2009. Genetic control of physiological traits associated to low temperature growth in sunflower under early sowing conditions. Plant Sci. 177:349-359.

Chang, C.L. and M.F. Sie. 2011. Fuzzy logic control approach for plant growth regulation system. Proc. 6th Intelligent Living Technology Conf. (2011 ILT), 1275-1282, 3 June 2011 [in Chinese].

Cook, S.K. 2009a. Sunflowers-A growers guide. Technique Report, ADAS UK Ltd., Surrey, UK, 2009. 5 Mar. 2009. <http://www.hgca.com/ document.aspx?fn=load\&media_id=5014\& publicationId $=0>$.

Cook, S.K. 2009b. Sunflower growth stages. Technique report. GB Seeds Ltd and HGCA, Suffolk, UK.

Engel, T., G. Hoogenboom, J.W. Jones, and P.W Wilkens. 1997. AEGIS/WIN: A computer program for the application of crop simulation models across geographic areas. Agron. J. 89: 919-928.

Fasanghari, M. and G.A. Montazer. 2010. Design and implementation of fuzzy expert system for Tehran stock exchange portfolio recommendation. Expert Syst. Appl. 37:6138-6147.

Jame, Y.W. and H.W. Cutforth. 1996. Crop growth models for decision support systems. Can. J. Plant Sci. 76:9-19.

Kolhe, S., R. Kamal, H.S. Saini, and G.K. Gupta. 2011. An intelligent multimedia interface for fuzzy-logic based inference in crops. Expert Syst. Appl. 38:14592-14601.

Kuo, Y.H., J.P. Hsu, and C.W. Wang. 1998. A parallel fuzzy inference model with distributed prediction scheme for reinforcement learning. IEEE Trans. Syst. Man Cyber. 28:160-172.

Marks II, R.J. 1994. Fuzzy logic technology and applications. IEEE Technology Update Series. p. 19-24.

Meyer, R., D. Belshe, and D. O’Brien. 1999. Sunflower cost-return prospects. Part of the High Plains Sunflower Production Handbook, MF-2384, Kansas State Univ. Agr. Experiment
Station and Cooperative Extension Service. p. $17-20$.

Monteith, J.L. 1996. The quest for balance in crop modeling. Agron. J. 88:695-697.

Putnam, D.H., E.S. Oplinger, D.R. Hicks, B.R. Durgan, D.M. Noetzel, R.A. Meronuck, J.D. Doll, and E.M. Schulte. 1990. Alternative field crops manual: Sunflower. 35. 5 Mar. 2011. $<$ http://www.hort.purdue.edu/newcrop/afcm/ sunflower.html>

Schneiter, A.A. and J.F. Miller. 1981. Description of sunflower growth stages. Crop Sci. 11:3-5.

Thornley, J.H.M. and J. France. 2007. Mathematical models in agriculture: Quantitative methods for the plant, animal and ecological sciences. 2nd Ed. CABI, Wallingford, UK.

Tu, K.Y., T.T. Lee, and W.J. Wang. 2000. Design of a multi-layer fuzzy logic controller for multiinput, multi-output systems. Fuzzy Sets Syst. 111:199-214.

Unger, P.W. 1986. Growth and development of irrigated sunflower in the Texas high plains. Agron. J. 78:507-515.

van Evert, F.K. and G.S. Campbell. 1994. A collection of object-oriented simulation models of agricultural systems. Agron. J. 86: 325-331.

van Evert, F.K., P.A. Leffelaar, M. Acutis, M. Adam, F. Ewert, H. van Keulen, and P. Trevisiol. 2007. Practical use of components in agro-ecological simulation. Proc. Summer Computer Simulation Conference (SCSC 2007), San Diego, CA, 16-19 July 2007.

Waterman, D.A. 1986. A Guide to expert systems, Addison-Wesley Longman Publishing, Boston, MA.

Whisler, F.D., B. Acock, D.N. Baker, R.E. Fye, H.F. Hodges, J.R. Lambert, H.E. Lemmon, J.M. McKinion, and V.R. Reddy. 1986. Crop simulation models in agronomic systems. Adv. Agron. 40:141-208.

Zadeh, L.A. 1965. Fuzzy sets. Inf. Control 8:338353.

Zadeh, L.A. 1972. A rationale for fuzzy control. J. Dyn. Syst.-T. ASME. 94:3-4. 


\section{Appendix A}

Rules database (growth stage fuzzy controller): There are 24 rules in total.

Rule 1 to Rule 8: If $X_{\mathrm{GH}}$ is $\mathrm{GH} n$ and $X_{\mathrm{GT}}$ is GTn. Then $Z_{\Omega}$ is STGn.

Rule 9 to Rule 16: If $X_{\mathrm{GH}}$ is $\mathrm{GH}$. Then $Z_{\Omega}$ is STGn.

Rule 17 to Rule 24: If $X_{\mathrm{GT}}$ is $\mathrm{GT}$. Then $Z_{\Omega}$ is $\mathrm{STG} n$.

Rules database (growth rate fuzzy controller): The following is an instance that presents the rule database of controller at 0 stage $(n=0)$. Different stage shows different control rules. What is in common is that the rule number in each controller is 29 .

Example:

Rule 1. If $X_{\mathrm{L}}$ is LL and $X_{\mathrm{U}}$ is UL. Then $Z_{\mathrm{GH}}$ is $\mathrm{GHH}$ and $Z_{\mathrm{GT}}$ is $\mathrm{GTH}$.

Rule 2. If $X_{\mathrm{L}}$ is $\mathrm{LL}$ and $X_{\mathrm{U}}$ is SL and $X_{\mathrm{T}}$ is TL. Then $Z_{\mathrm{GH}}$ is $\mathrm{GHH}$ and $Z_{\mathrm{GT}}$ is $\mathrm{GTH}$.

Rule 3. If $X_{\mathrm{L}}$ is $\mathrm{LL}$ and $X_{\mathrm{U}}$ is $\mathrm{UL}$ and $X_{\mathrm{T}}$ is TH. Then $Z_{\mathrm{GH}}$ is $\mathrm{GHH}$ and $Z_{\mathrm{GT}}$ is $\mathrm{GTH}$.

Rule 4. If $X_{\mathrm{L}}$ is $\mathrm{LL}$ and $X_{\mathrm{U}}$ is $\mathrm{UL}$ and $X_{\mathrm{W}}$ is TL. Then $Z_{\mathrm{GH}}$ is $\mathrm{GHH}$ and $Z_{\mathrm{GT}}$ is $\mathrm{GTH}$.

Rule 5. If $X_{\mathrm{L}}$ is $\mathrm{LL}$ and $X_{\mathrm{U}}$ is $\mathrm{UL}$ and $X_{\mathrm{W}}$ is TH. Then $Z_{\mathrm{GH}}$ is $\mathrm{GHH}$ and $Z_{\mathrm{GT}}$ is $\mathrm{GTH}$.

Rule 6. If $X_{\mathrm{L}}$ is $\mathrm{LL}$ and $X_{\mathrm{T}}$ is UL and $X_{\mathrm{W}}$ is TL. Then $Z_{\mathrm{GH}}$ is $\mathrm{GHH}$ and $Z_{\mathrm{GT}}$ is $\mathrm{GTH}$.

Rule 7. If $X_{\mathrm{L}}$ is LL and $X_{\mathrm{T}}$ is $\mathrm{UL}$ and $X_{\mathrm{W}}$ is TH. Then $Z_{\mathrm{GH}}$ is $\mathrm{GHH}$ and $Z_{\mathrm{GT}}$ is $\mathrm{GTH}$.

Rule 8. If $X_{\mathrm{L}}$ is LL and $X_{\mathrm{U}}$ is $\mathrm{UH}$ and $X_{\mathrm{T}}$ is TL. Then $Z_{\mathrm{GH}}$ is $\mathrm{GHH}$ and $Z_{\mathrm{GT}}$ is $\mathrm{GTH}$.

Rule 9. If $X_{\mathrm{L}}$ is $\mathrm{LL}$ and $X_{\mathrm{U}}$ is $\mathrm{UH}$ and $X_{\mathrm{W}}$ is TL. Then $Z_{\mathrm{GH}}$ is $\mathrm{GHH}$ and $Z_{\mathrm{GT}}$ is $\mathrm{GTH}$.

Rule 10. If $X_{\mathrm{L}}$ is $\mathrm{LL}$ and $X_{\mathrm{T}}$ is $\mathrm{TH}$ and $X_{\mathrm{W}}$ is TL. Then $Z_{\mathrm{GH}}$ is $\mathrm{GHH}$ and $Z_{\mathrm{GT}}$ is GTH.

Rule 11. If $X_{\mathrm{L}}$ is $\mathrm{LL}$ and $X_{\mathrm{U}}$ is $\mathrm{UL}$ and $X_{\mathrm{T}}$ is TL and $X_{\mathrm{W}}$ is WL. Then $Z_{\mathrm{GH}}$ is $\mathrm{GHH}$ and $Z_{\mathrm{GT}}$ is $\mathrm{GTH}$.

Rule 12. If $X_{\mathrm{L}}$ is $\mathrm{LM}$ and $X_{\mathrm{U}}$ is UM. Then $Z_{\mathrm{GH}}$ is $\mathrm{GHM}$ and $Z_{\mathrm{GT}}$ is GTM.

Rule 13. If $X_{\mathrm{L}}$ is $\mathrm{LM}$ and $X_{\mathrm{T}}$ is TM. Then $Z_{\mathrm{GH}}$ is $\mathrm{GHM}$ and $Z_{\mathrm{GT}}$ is GTM.

Rule 14. If $X_{\mathrm{L}}$ is $\mathrm{LM}$ and $X_{\mathrm{W}}$ is WM. Then $Z_{\mathrm{GH}}$ is $\mathrm{GHM}$ and $Z_{\mathrm{GT}}$ is GTM.

Rule 15. If $X_{\mathrm{L}}$ is $\mathrm{LM}$ and $X_{\mathrm{U}}$ is $\mathrm{UM}$ and $X_{\mathrm{W}}$ is WM. Then $Z_{\mathrm{GH}}$ is GHM and $Z_{\mathrm{GT}}$ is GTM.

Rule 16. If $X_{\mathrm{L}}$ is $\mathrm{LM}$ and $X_{\mathrm{T}}$ is $\mathrm{TM}$ and $X_{\mathrm{W}}$ is WM. Then $Z_{\mathrm{GH}}$ is GHM and $Z_{\mathrm{GT}}$ is GTM.

Rule 17. If $X_{\mathrm{L}}$ is $\mathrm{LM}$ and $X_{\mathrm{U}}$ is $\mathrm{UM}$ and $X_{\mathrm{T}}$ is TM. Then $Z_{\mathrm{GH}}$ is GHM and $Z_{\mathrm{GT}}$ is GTM.
Rule 18. If $X_{\mathrm{L}}$ is $\mathrm{LM}$ and $X_{\mathrm{U}}$ is $\mathrm{UM}$ and $X_{\mathrm{T}}$ is $\mathrm{TM}$ and $X_{\mathrm{W}}$ is $M^{n}$. Then $Z_{\mathrm{GH}}$ is GHM and $Z_{\mathrm{GT}}$ is GTM.

Rule 19. If $X_{\mathrm{L}}$ is $\mathrm{LH}$ and $X_{\mathrm{U}}$ is $\mathrm{UH}$. Then $Z_{\mathrm{GH}}$ is GHL and $Z_{\mathrm{GT}}$ is GTL.

Rule 20. If $X_{\mathrm{L}}$ is $\mathrm{LH}$ and $X_{\mathrm{U}}$ is $\mathrm{UH}$ and $X_{\mathrm{T}}$ is $\mathrm{TH}$. Then $Z_{\mathrm{GH}}$ is GHL and $Z_{\mathrm{GT}}$ is GTL.

Rule 21. If $X_{\mathrm{L}}$ is $\mathrm{LH}$ and $X_{\mathrm{U}}$ is $\mathrm{UH}$ and $X_{\mathrm{T}}$ is TL. Then $Z_{\mathrm{GH}}$ is GHL and $Z_{\mathrm{GT}}$ is GTL.

Rule 22. If $X_{\mathrm{L}}$ is $\mathrm{LH}$ and $X_{\mathrm{U}}$ is $\mathrm{UH}$ and $X_{\mathrm{W}}$ is WH. Then $Z_{\mathrm{GH}}$ is GHL and $Z_{\mathrm{GT}}$ is GTL.

Rule 23. If $X_{\mathrm{L}}$ is $\mathrm{LH}$ and $X_{\mathrm{U}}$ is $\mathrm{UH}$ and $X_{\mathrm{W}}$ is WL. Then $Z_{\mathrm{GH}}$ is GHL and $Z_{\mathrm{GT}}$ is GTL.

Rule 24. If $X_{\mathrm{L}}$ is $\mathrm{LH}$ and $X_{\mathrm{T}}$ is $\mathrm{TH}$ and $X_{\mathrm{W}}$ is WH. Then $Z_{\mathrm{GH}}$ is GHL and $Z_{\mathrm{GT}}$ is GTL.

Rule 25. If $X_{\mathrm{L}}$ is $\mathrm{LH}$ and $X_{\mathrm{T}}$ is $\mathrm{TH}$ and $X_{\mathrm{W}}$ is WL. Then $Z_{\mathrm{GH}}$ is GHL and $Z_{\mathrm{GT}}$ is GTL.

Rule 26. If $X_{\mathrm{L}}$ is $\mathrm{LH}$ and $X_{\mathrm{U}}$ is $\mathrm{UL}$ and $X_{\mathrm{T}}$ is $\mathrm{TH}$. Then $Z_{\mathrm{GH}}$ is GHL and $Z_{\mathrm{GT}}$ is GTL.

Rule 27. If $X_{\mathrm{L}}$ is $\mathrm{LH}$ and $X_{\mathrm{U}}$ is UL and $X_{\mathrm{W}}$ is WH. Then $Z_{\mathrm{GH}}$ is GHL and $Z_{\mathrm{GT}}$ is GTL.

Rule 28. If $X_{\mathrm{L}}$ is $\mathrm{LH}$ and $X_{\mathrm{T}}$ is TL and $X_{\mathrm{W}}$ is WH. Then $Z_{\mathrm{GH}}$ is GHL and $Z_{\mathrm{GT}}$ is GTL.

Rule 29. If $X_{\mathrm{L}}$ is $\mathrm{LH}$ and $X_{\mathrm{U}}$ is $\mathrm{UH}$ and $X_{\mathrm{T}}$ is $\mathrm{TH}$ and $X_{\mathrm{W}}$ is WH. Then $Z_{\mathrm{GH}}$ is $\mathrm{GHL}$ and $Z_{\mathrm{GT}}$ is $\mathrm{GTL}$.

Rules database (environmental factor fuzzy controller):

Example:

Rule 1. If $X_{\mathrm{L}}$ is LL and $X_{\mathrm{U}}$ is UL. Then $Z_{\mathrm{U}}$ is $\mathrm{UH}$.

Rule 2. If $X_{\mathrm{L}}$ is LL and $X_{\mathrm{U}}$ is $\mathrm{UL}$ and $X_{\mathrm{T}}$ is TL. Then $Z_{\mathrm{U}}$ is $\mathrm{UH}$ and $Z_{\mathrm{T}}$ is $\mathrm{TH}$.

Rule 3. If $X_{\mathrm{L}}$ is $\mathrm{LL}$ and $X_{\mathrm{U}}$ is $\mathrm{UL}$ and $X_{\mathrm{T}}$ is TH. Then $Z_{\mathrm{U}}$ is $\mathrm{UH}$ and $Z_{\mathrm{T}}$ is TL.

Rule 4. If $X_{\mathrm{L}}$ is LL and $X_{\mathrm{U}}$ is UL and $X_{\mathrm{W}}$ is WL. Then $Z_{\mathrm{U}}$ is $\mathrm{UH}$ and $Z_{\mathrm{W}}$ is WH.

Rule 5. If $X_{\mathrm{L}}$ is $\mathrm{LL}$ and $X_{\mathrm{U}}$ is $\mathrm{UL}$ and $X_{\mathrm{W}}$ is WH. Then $Z_{\mathrm{U}}$ is $\mathrm{UH}$ and $Z_{\mathrm{W}}$ is WL.

Rule 6. If $X_{\mathrm{L}}$ is $\mathrm{LL}$ and $X_{\mathrm{T}}$ is TL and $X_{\mathrm{W}}$ is WL. Then $Z_{\mathrm{T}}$ is $\mathrm{TH}$ and $Z_{\mathrm{W}}$ is $\mathrm{WH}$.

Rule 7. If $X_{\mathrm{L}}$ is $\mathrm{LL}$ and $X_{\mathrm{T}}$ is TL and $X_{\mathrm{W}}$ is WH. Then $Z_{\mathrm{T}}$ is $\mathrm{TH}$ and $Z_{W}$ is WL.
Rule 8. If $X_{\mathrm{L}}$ is $\mathrm{LL}$ and $X_{\mathrm{U}}$ is $\mathrm{UH}$ and $X_{\mathrm{T}}$ is TL. Then $Z_{\mathrm{U}}$ is $\mathrm{UL}$ and $Z_{\mathrm{T}}$ is TH.

Rule 9. If $X_{\mathrm{L}}$ is $\mathrm{LL}$ and $X_{\mathrm{U}}$ is $\mathrm{UH}$ and $X_{\mathrm{W}}$ is WL. Then $Z_{\mathrm{U}}$ is $\mathrm{UL}$ and $Z_{\mathrm{W}}$ is $\mathrm{WH}$.

Rule 10. If $X_{\mathrm{L}}$ is $\mathrm{LL}$ and $X_{\mathrm{T}}$ is $\mathrm{TM}$ and $X_{\mathrm{W}}$ is WL. Then $Z_{\mathrm{T}}$ is TL and $Z_{\mathrm{W}}$ is $\mathrm{WH}$.

Rule 11. If $X_{\mathrm{L}}$ is LL and $X_{\mathrm{U}}$ is $\mathrm{UL}$ and $X_{\mathrm{T}}$ is TL and $X_{\mathrm{W}}$ is WL. Then $Z_{\mathrm{U}}$ is $\mathrm{UH}$ and $Z_{\mathrm{T}}$ is $\mathrm{TH}$ and $Z_{\mathrm{W}}$ is $\mathrm{WH}$.

Rule 12. If $X_{\mathrm{L}}$ is $\mathrm{LM}$ and $X_{\mathrm{U}}$ is UM. Then $Z_{\mathrm{U}}$ is $\mathrm{UM}$.

Rule 13. If $X_{\mathrm{L}}$ is $\mathrm{LM}$ and $X_{\mathrm{T}}$ is TM. Then $Z_{\mathrm{T}}$ is TM.

Rule14. If $X_{\mathrm{L}}$ is $\mathrm{LM}$ and $X_{\mathrm{W}}$ is WM. Then $Z_{\mathrm{W}}$ is WM.

Rule 15. If $X_{\mathrm{L}}$ is $\mathrm{LM}$ and $X_{\mathrm{U}}$ is $\mathrm{UM}$ and $X_{W}$ is WM. Then $Z_{\mathrm{U}}$ is $\mathrm{UM}$ and $Z_{\mathrm{W}}$ is WM.

Rule 16. If $X_{\mathrm{L}}$ is $\mathrm{LM}$ and $X_{\mathrm{T}}$ is $M^{n}$ and $X_{\mathrm{W}}$ is $M^{n}$. Then $Z_{\mathrm{T}}$ is $\mathrm{TM}$ and $Z_{\mathrm{W}}$ is WM.

Rule 17. If $X_{\mathrm{L}}$ is $\mathrm{LM}$ and $X_{\mathrm{U}}$ is $\mathrm{UM}$ and $X_{\mathrm{T}}$ is $M^{n}$. Then $Z_{\mathrm{U}}$ is $\mathrm{UM}$ and $Z_{\mathrm{T}}$ is TM.

Rule 18. If $X_{\mathrm{L}}$ is $\mathrm{LM}$ and $X_{\mathrm{U}}$ is $\mathrm{UM}$ and $X_{\mathrm{T}}$ is $\mathrm{TM}$ and $X_{\mathrm{W}}$ is .WM Then $Z_{\mathrm{U}}$ is $\mathrm{UM}$ and $Z_{\mathrm{T}}$ is $\mathrm{TM}$ and $Z_{\mathrm{W}}$ is $\mathrm{WM}$.

Rule 19. If $X_{\mathrm{L}}$ is $\mathrm{LH}$ and $X_{\mathrm{U}}$ is $\mathrm{UH}$. Then $Z_{\mathrm{U}}$ is $\mathrm{UL}$.

Rule 20. If $X_{\mathrm{L}}$ is $\mathrm{LH}$ and $X_{\mathrm{U}}$ is $\mathrm{UH}$ and $X_{\mathrm{T}}$ is $\mathrm{TH}$. Then $Z_{\mathrm{U}}$ is $\mathrm{UL}$ and $Z_{\mathrm{T}}$ is $\mathrm{TL}$.

Rule 21. If $X_{\mathrm{L}}$ is $\mathrm{LH}$ and $X_{\mathrm{U}}$ is $\mathrm{UH}$ and $X_{\mathrm{T}}$ is $\mathrm{TL}$. Then $Z_{\mathrm{U}}$ is $\mathrm{UL}$ and $Z_{\mathrm{T}}$ is $\mathrm{TH}$.

Rule 22. If $X_{\mathrm{L}}$ is $\mathrm{LH}$ and $X_{\mathrm{U}}$ is $\mathrm{UH}$ and $X_{\mathrm{W}}$ is $\mathrm{WH}$. Then $Z_{\mathrm{U}}$ is $\mathrm{UL}$ and $Z_{\mathrm{W}}$ is $\mathrm{WL}$.

Rule 23. If $X_{\mathrm{L}}$ is $\mathrm{LH}$ and $X_{\mathrm{U}}$ is $\mathrm{UH}$ and $X_{\mathrm{W}}$ is $\mathrm{WL}$. Then $Z_{\mathrm{U}}$ is $\mathrm{UL}$ and $Z_{\mathrm{W}}$ is $\mathrm{WH}$.

Rule 24. If $X_{\mathrm{L}}$ is $\mathrm{LH}$ and $X_{\mathrm{T}}$ is $\mathrm{TH}$ and $X_{\mathrm{W}}$ is $\mathrm{WH}$. Then $Z_{\mathrm{T}}$ is $\mathrm{TL}$ and $Z_{\mathrm{W}}$ is $\mathrm{WL}$

Rule 25. If $X_{\mathrm{L}}$ is $\mathrm{LH}$ and $X_{\mathrm{T}}$ is $\mathrm{TH}$ and $X_{\mathrm{W}}$ is WL. Then $Z_{\mathrm{T}}$ is TL and $Z_{\mathrm{W}}$ is $\mathrm{WH}$.

Rule 26. If $X_{\mathrm{L}}$ is $\mathrm{LH}$ and $X_{\mathrm{U}}$ is $\mathrm{UL}$ and $X_{\mathrm{T}}$ is TH. Then $Z_{\mathrm{U}}$ is $\mathrm{UH}$ and $Z_{\mathrm{T}}$ is TL.

Rule 27. If $X_{\mathrm{L}}$ is $\mathrm{LH}$ and $X_{\mathrm{U}}$ is UL and $X_{\mathrm{W}}$ is WH. Then $Z_{\mathrm{U}}$ is $\mathrm{UH}$ and $Z_{W}$ is $\mathrm{WL}$.

Rule 28. If $X_{\mathrm{L}}$ is $\mathrm{LH}$ and $X_{\mathrm{T}}$ is TL and $X_{\mathrm{W}}$ is WH. Then $Z_{\mathrm{T}}$ is $\mathrm{TH}$ and $Z_{\mathrm{W}}$ is WL.

Rule 29. If $X_{\mathrm{L}}$ is $\mathrm{LH}$ and $X_{\mathrm{U}}$ is $\mathrm{UH}$ and $X_{\mathrm{T}}$ is $\mathrm{TH}$ and $X_{\mathrm{W}}$ is WH. Then $Z_{\mathrm{U}}$ is $\mathrm{UL}$ and $Z_{\mathrm{T}}$ is $\mathrm{TL}$ and $Z_{\mathrm{W}}$ is $\mathrm{WL}$. 